\title{
Diversity of pimpline wasps (Hymenoptera: Ichneumonidae) in the Central highlands of Vietnam
}

\author{
Đa dạng các loài ong cự phân ho Pimplinae (Hymenoptera: Ichneumonidae) ờ \\ khu vục Tây Nguyên \\ Short communication
}

Pham, Thi Nhi*

Institute of Ecology and Biological Resources, Vietnam Academy of Science and Technology, 18 Hoang Quoc Viet, Hanoi, Vietnam

\begin{abstract}
In this paper, 48 ichneumonid species of 14 genera, two tribes of the subfamily Pimplinae were listed on the basis of material collected during the time from 2003 to 2011 in four provinces from the Central Highlands of Vietnam and a lesser degree of previous records. Among 48 aforemention pimpline no less than 16 species were described recently as new for science. The diversity of topography and vegetation types resulted in the high level of species richness and new discoveries of the region.
\end{abstract}

Bài báo này giới thiệu danh sách 48 loài ong cụ thuộc 14 giống, 2 tộc của phân họ Pimplinae dựa trên việc phân tích các mẫu vật thu tại Tây Nguyên trong thời gian tù năm 2003-2011 và một số it loài dựa trên các ghi nhận trước đây. Trong số đó, 16 loài mới được mô tả trong thời gian 5 năm trở lại đây. Sụ đa dạng về địa hình và các kiểu thảm thực vật khác nhau đã tạo nên tính đa dạng thành phần loài và các phát hiện mói của ong cự ở khu vực Tây Nguyên đầy tiềm năng này.

Keywords: ichneumonid, parasitoid, diversity

\section{Introduction}

Located in the south of Truong Son Range, Central Highlands of Vietnam is recognized as one of centers of biodiversity of the country. The complex topography of the area with many plateaus over $1,000 \mathrm{~m}$ above level sea (Play Ku, Dak Lak, Da Lat, Chu Yang Sin and Di Linh Plateaus) and divided by several large rivers (Se San River, Ba River, Sere Pok River) has given a wide variety of vegetation types and microclimate conditions. Therefore, this region is home to a high level of biodiversity and may be a focal point or hotspot of endemism within mainland Southeast Asia (Sterling et al., 2006).

Pimplinae is a moderately large group of Ichneumonidae (Hymenoptera) comprising more than 1500 species classified in 77 genera. The members of this subfamily are either idiobiont endoparasitoids of Lepidoptera; idiobiont ectoparasitoids of xylophagous Coleoptera larvae or koinobiont ectoparasitoids of spiders. As a consequence, it is one of the most extensively studied ichneumonid taxa, both from a taxonomic perspective and from a biological one (Gauld et al., 2002; Yu et al., 2005; Gauld \& Dubois, 2006; Palacio et al., 2007, Pham, 2013).

In Vietnam, Pimplinae has been investigated more comprehensively with a total of 122 species recorded in 21 genera (Pham, 2013). In this paper, the author would like to summary the diversity of Pimplinae from the Central Highlands of Vietnam and emphasize the potential of biological resources of the region.

\section{Materials and methods}

Taxonomic decision in this paper was based on material collected from 2003 to 2011 in Kon Tum, Gia Lai, Dak Lak and Lam Dong provinces and a lesser degree on previous records of foreign authors.

Abbreviations of some museums where the types are being deposited as below: 

AEIC
BMNH
USA, Florida, Gainesville, American Entomological Institute
BPBM
UK, London, Natural History Museum
CNC
USA, Hawaii, Honolulu, Bernice P. Bishop Museum
GPTA
HNHM
Canada, Ontario, Ottawa, Canadian National Collection of Insects
IEBR
India, Delhi, Delhi University, Department of Zoology, Gupta Collection
OUMNH
TARI
USNM
ZFMK
ZMHB
ZMUC
Hungary, Budapest, Hungarian Natural History Museum
Vietnam, Hanoi, Institute of Ecology and Biological Resources
United Kingdom, Oxford, University Museum of Natural History
Taiwan, Taichung, Taiwan Agricultural Research Institute
USA, Washington D.C., National Museum of Natural History
Germany, Bonn, Zoological Research Museum Alexander Koenig
Germany, Berlin, Museum für Naturkunde der Humboldt-Universität
Denmark, Copenhagen, University of Copenhagen, Zoological Museum
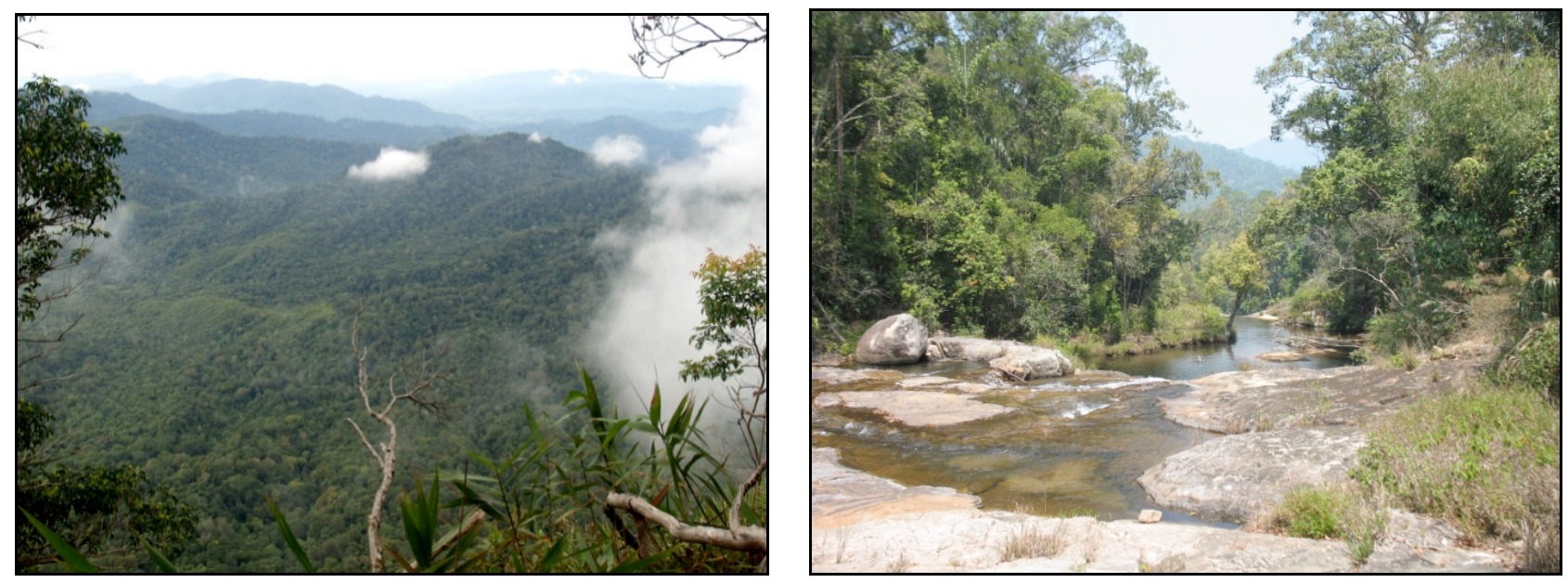

Figure 1. Evergreen forests of Chu Mom Ray NP in Kon Tum Province (left) and Chu Yang Sin NP in Dak Lak Province (right). Photographs by T. N. Vu and T. V. Hoang

\section{Results}

A total of 48 species of 14 genera, two tribe of the subfamily Pimplinae were recorded from the Central Highlands of Vietnam. Of which, 16 species were described as new for science on the basis of material collected from this region. In addition, the unique male specimen of the genus Pimpla will be described in near future. Among the region, Chu Yang Sin NP is a valuable part of the network of protected areas in Vietnam with 10 newly discovered pimpline species, only lesser than Hoang Lien NP in the Northwest (12 new species). Beside the newly described species, five other species have been documented from the country but restricted only in the Central Highlands area (Pham, 2013). Resulted from many newly recently described and recorded species, the species composition of Pimplinae in the Central Highlands is quite different to those of other geographical regions. In compared with other geographic regions of Vietnam, the species richness of Pimplinae is similar to the North Central of Vietnam and only less than the northwestern region but the species composition is relatively different.

\section{PIMPLINAE Wesmael, 1845 Tribe Ephialtini \\ The Camptotypus genus-group \\ Camptotypus Kriechbaumer, 1889}

Camptotypus Kriechbaumer, 1889: 311. Type-species: Camptotypus sellatus Kriechbaumer, by subsequent designation, Viereck, 1914: 27.

\section{Camptotypus trui PHAM, BROAD \& WÄGELE, 2012}

Camptotypus trui Pham, Broad \& Wägele, 2012. Dtsch. Entomol. Z. 59 (1): 134. Holotype: , , Vietnam: Kon Tum, Chu Mom Ray NP (IEBR).

Distribution: Vietnam (Kon Tum and Nghe An) (Pham et al., 2012b).

\section{The Sericopimpla genus group}

\section{Acrodactyla Haliday, 1838}

Barypus Haliday in Curtis, 1837: 37. Type-species: Barypus degener Haliday by subsequent designation (Westwood, 1840). Junior homonym of Barypus Laporte, 1835.

Acrodactyla Haliday, 1838: 117. Replacement name for Barypus Haliday.

\section{Acrodactyla lachryma PHAM, BROAD, MATSUMOTO \& BöHME, 2012}

Acrodactyla lachryma Pham, Broad, Matsumoto \& Böhme, 2012. Zootaxa, 3207: 6. Holotype: ㅇ, Vietnam: Kontum, Chu Mom Ray NP (RMNH).

Distribution: Vietnam (Kon Tum) (Pham et al., 2012a).

\section{Acropimpla Townes, 1960}

Selenaspis Roman, 1910: 191. Name preoccupied by Bleeker, 1858 and by Leonardi, 1898. Type: Hemipimpla alboscutellaris Szepligeti; original designation.

Acropimpla Townes, 1960: 159. Type species: Charitopimpla leucostoma Cameron; original designation. 


\section{Acropimpla lampei PHAM, BROAD \& WÄGELE, 2011}

Acropimpla lampei Pham, Broad, Wägele, 2011. Zootaxa, 2921: 6. Holotype: ( (IEBR), Dak Lak, Ea So NR. Distribution: Vietnam (Dak Lak) (Pham et al., 2011d).

\section{Acropimpla leucostoma (CAMERON, 1907)}

Charitopimpla leucostoma Cameron, 1907. Tijdschr. Ent., 50: 97. Holotype: ㅇ, India: Sikkim (BMNH).

Acropimpla leucostoma: Townes \& Townes (1960).

Distribution: Vietnam (Vinh Phuc, Lai Chau, Phu Tho, Hoa Binh, Ninh Binh, Ha Tinh, Thua Thien-Hue, Kon Tum, Dak Lak and Dong Nai), China, India, Indonesia, Japan, Laos, Myanmar and Sri Lanka (Yu et al., 2005; Pham, 2013).

Chablisea Gauld \& Dubois, 2006

Chablisea Gauld \& Dubois, 2006: 544. Type-species: Chablisea imbiba, by original designation.

\section{Chablisea khuatdanglongi PHAM, BROAD, MATsOMOTO} \& WÄGELE, 2011

Chablisea khuatdanglongi Pham, Broad, Matsomoto \& Wägele, 2011. Biologia 66/6: 1136. Holotype: ${ }^{\circ}$, Vietnam: Hanoi, Thach That, Tan Xa (IEBR).

Distribution: Vietnam (Hanoi, Hoa Binh, Ninh Binh, Kon Tum and Dak Lak provinces) (Pham et al., 2011b).

Sericopimpla Kriechbaumer, 1895

Sericopimpla Kriechbaumer, 1895: 135. Type-species: Pimpla sericata Kriechbaumer, 1895, by monotypy.

\section{Sericopimpla sagrae (VOLLENHOVEN, 1879)}

Pimpla sagrae Vollenhoven, 1879. Stettin. Ent. Ztg., 40: 149. Lectotype: + , Indonesia: Java (RMNH).

Sericopimpla sagrae: Townes, Townes \& Gupta (1961). Distribution: Vietnam (Thai Nguyen, Phu Tho, Ha Noi, Hoa Binh, Nghe An, Ha Tinh, Thua Thien-Hue, Dak Lak, Dong Nai), China, India, Indonesia, Malaysia, Philippines, Sri Lanca, Taiwan, Japan, and Korea ( $\mathrm{Yu}$ et al., 2005; Pham et al., 2011e).

\section{Zaglyptus Foerster, 1869}

Zaglyptus Foerster, 1869. Verh. Naturh. Ver. Rheinlande 25: 166. Type-species: Polysphincta varipes Gravenhorst, by subsequent designation, Woldstedt, 1877: 17.

\section{Zaglyptus guptai PHAM, BROAD \& WÄGELE, 2013}

Zaglyptus guptai Pham, Broad \& Wägele, 2013. Zootaxa, 3702 (5): 486. Holotype: , Vietnam: Dak Lak, Chu Yang Sin NP (RMNH).

Distribution: Vietnam (Dak Lak) (Pham et al., 2013c).

\section{Zaglyptus nigrolineatus GUPTA, 1961}

Zaglyptus nigrolineatus Gupta, 1961.

Lissopimpla Kriechbaumer, 1889: 309. Type-species: Lissopimpla octoguttata Kriechbaumer, 1899 (= Pimpla excelsa Costa), by subsequent designation, Ashmead, 1900: 55.

Distribution: Vietnam (Dak Lak Province), India (Yu et al., 2005; Pham et al., 2013c).

Tribe Pimplini

The Pimpla genus-group

Echthromorpha Holmgren, 1868

Echthromorpha Holmgren, 1868: 406. Type-species:

Echthromorpha maculipennis Holmgren, 1868.

\section{Echthromorpha agrestoria (SWEDERUS, 1787)}

Ichneumon agrestorius Swederus, 1787. Kongliga Svenska Vetenskapsakademiens Handlingar. 8: 279. Holotype: o, Tahiti (BMNH).

Distribution: Vietnam (Ha Giang, Son La, Thai Nguyen, Vinh Phuc, Hanoi, Phu Tho, Hoa Binh, Ninh Binh, Thanh Hoa, Nghe An, Ha Tinh, Quang Binh, Thua Thien-Hue, Da Nang, Kon Tum and Dong Nai provinces), Australia, Bangladesh, Cameroon, China, Democratic Republic of Congo, Egypt, Ethiopia, Fiji, Guam, Guinea, India, Indonesia, Japan, Kenya, Laos, Liberia, Madagascar, Malaysia, Mauritius, Mozambique, Myanmar, New Caledonia, Palau, Papua New Guinea, the Philippines, Réunion, Senegal, Sierra Leone, Singapores, Society Islands, South Africa, Sri Lanca, Sudan, Tanzania, Thailand, Togo, U.S.A, Uganda, Vanuatu, Zimbabwe (Yu et al., 2005; Pham, 2013).

\section{Lissopimpla Kriechbaumer, 1889}

Lissopimpla Kriechbaumer, 1889: 309. Type-species: Lissopimpla octoguttata Kriechbaumer, 1899 (= Pimpla excelsa Costa), by subsequent designation, Ashmead, 1900: 55.

\section{Lissopimpla basalis (VOLLENHOVEN, 1879)}

Pimpla basalis Vollenhoven, 1879. Stettin Ent. Ztg. 40 (4-6): 148. Holotype: $\widehat{\jmath}$, Indonesia: Sumatra (RMNH). Lissopimpla basalis: Townes, Townes \& Gupta (1961). Distribution: Vietnam (Hoa Binh, Ninh Binh, Nghe An, Ha Tinh, Thua Thien-Hue, Quang Nam, Dak Lak), Indonesia and the Philippines (Yu et al., 2005; Pham, 2013).

Pimpla Fabricius, 1804

Pimpla Fabricius, 1804: 112. Type-species: Ichneumon instigator Fabricius (= Ichneumon hypochondriaca Retzius), by subsequent designation (Opinion 159, International Commission on Zoological Nomenclature, 1954: 282).

\section{Pimpla chuyangsinensis PHAM, BROAD, DANG \& BÖHME, 2013}

Pimpla chuyangsinensis Pham, Broad, Dang \& Böhme, 2013. Org. Divers. Evol. 13(3): 401. Holotype: $\widehat{\jmath}$, Vietnam: Dak Lak, Chu Yang Sin NP (RMNH).

Distribution. Vietnam (Dak Lak) (Pham et al., 2013a).

\section{Pimpla flavipalpis CAMERON, 1899}

Pimpla flavipalpis Cameron, 1899. Mem. \& Proc. Manchester Lit. Phil. Soc., 43 (3): 174. Lectotype: + , India: Meghalaya: Khasi Hill (BMNH).

Distribution: Vietnam (Lao Cai, Kon Tum), China, India, Myanmar, Nepal and Taiwan (Yu et al., 2005; Pham et al. 2013a).

\section{Pimpla sp.}

Remarks: One male specimen collected in Bidoup Nui Ba National Park, Lam Dong Province in 2005 may reveal a new species. It will be critically examined in near future.

Xanthopimpla Saussure, 1892

Xanthopimpla Saussure 1892: 13. Type-species: Xanthopimpla hova Saussure, by subsequent designation, Ashmead, 1900: 56. 


\section{Xanthopimpla alternans KRIEGER, 1914}

Xanthopimpla alternans Krieger, 1914. Arch. f. Naturgesch., (A) 80 (6): 31. Holotype: +, Taiwan: Chiayi (ZMHB).

Distribution: Vietnam (Nghe An, Nha Trang, Gia Lai), China, India, Indonesia, Malaysia, Sri Lanka and Taiwan (Yu et al., 2005; Pham, 2013).

\section{Xanthopimpla atriclunis TOWNES \& CHIU, 1970}

Xanthopimpla atriclunis Townes \& Chiu, 1970. Mem. Amer. Ent. Inst., 14: 157. Holotype: 9 , the Philippines: Alcate, Victoria, Mindoro Oriental (AEIC).

Distribution: Vietnam (Thua Thien-Hue, Dak Lak and Dong Nai provinces), and the Philippines (Yu et al., 2005; Pham et al., 2011c).

\section{Xanthopimpla boehmei PHAM, BROAD, MATSUMOTO \& WÄGELE, 2011}

Xanthopimpla boehmei Pham, Broad, Matsumoto \& Wägele, 2011. Zootaxa, 3056: 41. Holotype: \&, Vietnam: Dak Lak, Chu Yang Sin NP (IEBR).

Distribution: Vietnam (Dak Lak) (Pham et al., 2011c).

\section{Xanthopimpla calva TOWNES \& CHIU, 1970}

Xanthopimpla calva Townes \& Chiu, 1970. Mem. Amer. Ent. Inst., 14: 240. Holotype: + , the Philippines: Gapan, Nueva Ecija (AEIC).

Distribution: Vietnam (Nghe An,Dak Lak), India, Malaysia, Myanmar Philippines ( $\mathrm{Yu}$ et al., 2005; Pham et al., 2011c).

\section{Xanthopimpla chiuae PHAM, BROAD, MATSUMOTO \& WÄGELE, 2011}

Xanthopimpla chiuae Pham, Broad, Matsumoto \& Wägele, 2011. Zootaxa, 3056: 22. Holotype: \&, Vietnam: Dak Lak, Chu Yang Sin NP (RMNH).

Distribution: Vietnam (Dak Lak) (Pham et al., 2011c).

\section{Xanthopimpla clivulus TOWNES \& CHIU, 1970}

Xanthopimpla clivulus Townes \& Chiu, 1970. Mem. Amer. Ent. Inst., 14: 160. Holotype: , Indonesia: Java (AEIC).

Distribution: Vietnam (Thai Nguyen, Vinh Phuc, and Dak Lak), India, Indonesia, Malaysia and Singapore (Yu et al., 2005; Pham et al., 2011c).

\section{Xanthopimpla curvimaculata (CAMERON, 1899)}

Pimpla curvimaculata Cameron, 1899. Mem. \& Proc. Manchester Lit. Phil. Soc. 43 (3): 158. Holotype: ふૈ, India: Khasi Hills in Assam (OUMNH).

Xanthopimpla cuvimaculata: Krieger (1914).

Distribution: Vietnam (Phu Tho, Dak Lak and Dong Nai), India, Malaysia and Thailand (Yu et al., 2005; Pham et al., 2011c).

\section{Xanthopimpla elegans (VOLLENHOVEN, 1879)}

Pimpla elegans Vollenhoven, 1879. Stettin. Ent. Ztg. 40: 147. Holotype: 9 , Indonesia: Java (RMNH).

Xanthopimpla elegans: Krieger (1914).

Distribution: Vietnam (Phu Tho, Ninh Binh, Nghe An, Ha Tinh, Quang Tri, Thua Thien-Hue, Kon Tum, Dak Lak, Lam Dong, Dong Nai), India, Indonesia, Laos, Malaysia, Myanmar, Nepal, Singapore, Sri Lanka, Taiwan, Thailand and the Philippines (Yu et al., 2005; Pham et al., 2011c).

\section{Xanthopimpla fastigiata KRIEGER, 1914}

Xanthopimpla fastigiata Krieger, 1914. Arch. f. Na-

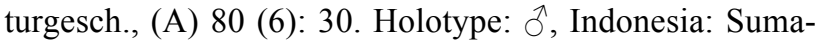
tra, Sarik (ZMHB).

Distribution: Vietnam (Phu Tho, Hanoi, Thua Thien-Hue and Dak Lak), India, Indonesia, Malaysia, Singapore, Thailand and the Philippines (Yu et al., 2005; Pham et al., 2011c).

\section{Xanthopimpla hienae PhAM, BRoAd, Matsumoto \& WÄGELE, 2011}

Xanthopimpla hienae Pham, Broad, Matsumoto \& Wägele, 2011. Zootaxa, 3056: 35. Holotype: q, Vietnam: Dak Lak, Ea So NR (IEBR).

Distribution: Vietnam (Dak Lak) (Pham et al., 2011c).

\section{Xanthopimpla jacobsoni KRIEGER, 1914}

Xanthopimpla jacobsoni Krieger, 1914. Arch. f. Naturgesch. (A) 80 (6): 32. Holotype: q, Java: Semarang (HNHM).

Distribution: Vietnam (Dak Lak), Indonesia, Malaysia, New Guinea, Singapore, Sri Lanka, Thailand and the Philippines (Yu et al., 2005; Pham et al., 2011c).

\section{Xanthopimpla naenia MORLEY, 1913}

Xanthopimpla naenia Morley, 1913. Faun. British India, Hymenoptera, 3(1): 115. Holotype: 9 , India (OUMNH).

Distribution: Vietnam (Lam Dong), India, Malaysia, Japan, Taiwan and the Philippines (Townes \& Chiu, 1970; Yu et al., 2005).

\section{Xanthopimpla nana SCHULZ, 1906}

Xanthopimpla parva Cameron, 1905. Spolia Zeylanica 3: 136. Holotype: §̃, Sri Lanka: Peradeniya (BMNH). Junior homonym of Xanthopimpla parva Krieger, 1899.

Xanthopimpla nana Schulz, 1906. Spolia Hymenopterologica: 114. Replacement name.

Distribution: Vietnam (Thai Nguyen, Phu Tho, Hanoi, Hoa Binh, Ninh Binh, Nghe An, Lam Dong, Khanh Hoa and Dong Nai), Cambodia, China, India, Indonesia, Nepal, Sri Lanka, Taiwan, Thailand and the Philippines (Yu et al., 2005; Pham et al., 2011c).

\section{Xanthopimpla nigritarsis CAMERON, 1903}

Xanthopimpla nigritarsis Cameron, 1903. Jour. Straits Branch Roy. Asiatic Soc. 39: 138. Lectotype: 옹 Malaysia: Sarawak, Kuching (BMNH).

Distribution. Vietnam (Nghe An, Kon Tum and Dong Nai), India, Indonesia, Malaysia and the Philippines (Yu et al., 2005; Pham et al., 2011c).

\section{Xanthopimpla ochracea (SMITH, 1858)}

Pimpla ochracea Smith, 1858. Jour. Proc. Linn. Soc. London Zool., 4: 172. Holotype: + Indonesia: Molucca Island, Aru (OUMNH).

Xanthopimpla ochracea: Krieger (1899).

Distribution: Vietnam (Lai Chau, Ninh Binh, Phu Tho and Dak Lak), Australia, China, India, Indonesia, Myanmar, Papua New Guinea, Taiwan, Thailand, and the Philippines (Yu et al., 2005; Pham et al., 2011c).

Xanthopimpla pulvinaris TOWNES \& CHIU, 1970 
Xanthopimpla pulvinaris Townes \& Chiu, 1970. Mem. Amer. Ent. Inst., 14: 271. Holotype: + , Taiwan: Shinchu (TARI).

Distribution: Vietnam (Phu Tho, Dak Lak), China, Laos, Malaysia, Taiwan and Thailand (Yu et al., 2005; Pham et al., 2011c).

\section{Xanthopimpla pseudosternata PHAM, BROAD,} MATSUMOTO \& WÄGELE, 2011

Xanthopimpla pseudosternata Pham, Broad, Matsumoto \& Wägele, 2011. Zootaxa, 3056: 24. Holotype: \&, Vietnam: Dak Lak, Chu Yang Sin NP (RMNH).

Distribution: Vietnam (Dak Lak) (Pham et al., 2011c).

\section{Xanthopimpla punctata (FABRICIUS, 1781)}

Ichneumon punctatus Fabricius, 1781. Species Insectorum, 1: 437. Holotype: $\widehat{\jmath}$, India: Coromadel (ZMUC). Xanthopimpla punctata: Krieger (1899).

Distribution: Vietnam (entire country), Afghanistan, Australia, China, India, Indonesia, Japan, Pakistan, Bangladesh, Guam, Laos, Malaysia, Mauritius, Myanmar, Nepal, Nigeria, Papua New Guinea, Russia, Sri Lanka, Singapore, Taiwan, Thailand, the Philippines and Togo (Townes \& Chiu, 1970; Plant Protection Research Institute, 1976; Pham, 1997; Yu et al., 2005; Pham \& Le, 2007; Pham et al., 2011c).

\section{Xanthopimpla quatei TOWNES \& CHIU, 1970}

Xanthopimpla quatei Townes \& Chiu, 1970. Mem. Amer. Ent. Inst., 14: 150. Holotype: , Vietnam: $30 \mathrm{~km}$ north of Pleiku [now in Gia Lai Province], South Vietnam (BPBM).

Distribution: Vietnam (Gia Lai), Malaysia (Townes \& Chiu, 1970; Yu et al., 2005).

\section{Xanthopimpla regina MORLEY, 1913}

Xanthopimpla regina Morley, 1913. Faun. British India, Hymenoptera, 3 (1): 118. Holotype: 9 , East Pakistan: Sylhet (BMNH).

Distribution: Vietnam (Bac Can, Thai Nguyen, Vinh Phuc, Phu Tho, Hoa Binh, Ninh Binh and Dak Lak), Bangladesh, China, India, Indonesia, Laos, Malaysia, Myanmar, Nepal, Pakistan, Singapore, Taiwan and Thailand (Yu et al., 2005; Pham et al., 2011c).

\section{Xanthopimpla sexlineata CAMERON, 1907}

Xanthopimpla sexlineata Cameron, 1907. Tijdschr. v. Ent. 50: 103. Holotype: $\curvearrowright$, India: Sikkim (BMNH).

Distribution: Vietnam (Kon Tum), India, Thailand (Yu et al., 2005; Pham et al., 2011c).

Xanthopimpla spicula PHAM, BROAD, MATsUMOTO \& WÄGELE, 2011

Xanthopimpla spicula Pham, Broad, Matsumoto \& Wägele, 2011. Zootaxa, 3056: 12. Holotype: \&, Vietnam: Dak Lak Province, Ea So NR (IEBR).

Distribution: Vietnam (Dak Lak) (Pham et al., 2011c).

Xanthopimpla trias TOWNES \& CHIU, 1970

Xanthopimpla trias Townes \& Chiu, 1970. Mem. Amer. Ent. Inst., 14: 242. Holotype: $q$, India: Mercara, Mysore (GPTA).

Distribution: Vietnam (Dak Lak, Vinh Phuc provinces), China, India, Nepal, Taiwan and Thailand (Yu et al., 2005; Pham et al., 2011c).

Xanthopimpla tricapus TOWNES \& CHIU, 1970
Xanthopimpla tricapus Townes \& Chiu, 1970. Mem. Amer. Ent. Inst., 14: 260.

Xanthopimpla tricapus impressa Townes \& Chiu, 1970. Mem. Amer. Ent. Inst., 14: 260. Holotype: + , Myanmar: Toungoo, Karenni (ZMHB).

Distribution: Vietnam (Vinh Phuc, Nghe An, Dak Lak and Dong Nai provinces), India, Indonesia, Malaysia, Myanmar, Thailand and the Philippines (Yu et al., 2005; Pham et al., 2011c).

\section{Xanthopimpla varimaculata CAMERON, 1907}

Xanthopimpla varimaculata Cameron, 1907. Tijdschr. v. Ent., 50: 103. Holotype: ग̃, India: Sikkim (BMNH).

Distribution: Vietnam (Vinh Phuc, Hoa Binh, Nghe An, Ha Tinh, Thua Thien-Hue, Dak Lak, Dong Nai provinces) China, India (Yu et al., 2005; Pham et al., 2011).

\section{The Theronia genus-group}

Augerella Gupta, 1962

Theronia (Augerella) Gupta, 1962: 86. Type: Theronia (Augerella) orientalis orientalis Gupta; by original designation.

Augerella Gupta: Gupta (1987).

\section{Augerella achterbergi PHAM, BROAD \& LAMPE, 2011}

Augerella achterbergi Pham, Broad \& Lampe, 2011. Zootaxa, 2745: 68. Holotype: o, Vietnam: Dak Lak, Chu Yang Sin NP (RMNH).

Distribution: Vietnam (Dak Lak) (Pham et al., 2011a).

\section{Augerella vriesi PHAM, BROAD \& LAMPE, 2011}

Augerella vriesi Pham, Broad \& Lampe, 2011. Zootaxa, 2745: 68. Holotype: + , Vietnam: Dak Lak, Chu Yang Sin NP (RMNH).

Distribution: Vietnam (Dak Lak) (Pham et al., 2011a).

\section{Nomosphecia Gupta, 1962}

Theronia (Nomosphecia) Gupta, 1962. Pac. Ins. Mon. 4: 68. Type: Theronia (Nomosphecia) zebroides zebroides Krieger, by original designation.

Nomosphecia Gupta: Gupta (1987).

\section{Nomosphecia scutellata (GUPTA, 1962)}

Theronia (Nomosphecia) scutellata Gupta, 1962. Pac. Ins. Mon. 4: 81.

Theronia (Nomosphecia) scutellata scutellata Gupta, 1962. Pac. Ins. Mon. 4: 82. Holotype: @, Singapore (USNM).

Nomosphecia scutellata scutellata: Gupta (1987).

Distribution: Vietnam (Ninh Binh, Dak Lak and Dong Nai provinces), Malaysia, Singapore and the Philippines (Gupta, 1962; Yu et al., 2005; Pham et al. 2013b).

\section{Nomosphecia zebroides (KRIEGER, 1906)}

Theronia zebroides Krieger, 1906. Ztschr. Syst. Hymen. Dipt. 6: 236. Holotype: O, Indonesia: Sumatra (ZMHB).

Nomosphecia zebroides: Gupta (1987).

Distribution: Vietnam (Vinh Phuc, Phu Tho, Nghe An, Quang Binh, Quang Tri, Dak Lak, Dong Nai provinces), India, Indonesia, Japan, Malaysia and Taiwan (Gupta, 1962; Yu et al., 2005; Pham et al., 2013b).

\section{Parema Gupta, 1962}


Theronia (Parema) Gupta, 1962: 54. Type species: Theronia (Parema) nigrobalteata nigrobalteata Gupta; by original designation.

Parema Gupta: Gupta (1987).

\section{Parema nigrobalteata (CAMERON, 1899)}

Theronia nigrobalteata Cameron, 1899. Mem. \& Proc. Manchester Lit. Phil. Soc. 43 (3): 153. Holotype: , India: Khasi Hills, Assam (OUMNH).

Theronia (Parema) nigrobalteara Cameron: Gupta (1962).

Parema nigrobalteata (Cameron): Gupta (1987).

Distribution: Vietnam (Tuyen Quang, Phu Tho, Vinh Phuc, Bac Giang, Hoa Binh, Ninh Binh, Nghe An, Ha Tinh, Thua Thien-Hue, Quang Nam, Kon Tum, Dak Lak, Ninh Thuan, Dong Nai provinces), India, Indonesia, Malaysia, Myanmar, Singapore, Thailand and the Philippines (Gupta, 1962; Yu et al., 2005; Pham et al., 2013b).

\section{Theronia Holmgren, 1859}

Theronia Holmgren, 1859. Öfvers. Svenska Vetensk. Akad. Förh. 16: 123. Type-species: Pimpla flavicans Fabricius $=$ atalantae Poda, by monotypy.

\section{Theronia clathrata KRIEGER, 1899}

Theronia clathrata Krieger, 1899. Naturf. Gesell. Leipzig, Sitzber. 1897/98: 111. Holotype: 9 : India: Khasi Hills, Assam (ZMHB).

Theronis clathrata malayensis Gupta, 1962. Pac. Ins. Mon. 4: 50. Holotype: + : Malaysia Peninsula: Fraser's Hill, Pahang (BMNH).

Distribution: Vietnam (Ha Tinh, Dak Lak provinces), China, India, Indonesia, Malaysia, Sri Lanka, Taiwan and the Philippines (Gupta, 1962; Yu et al., 2005; Pham et. al. 2013b).

Theronia ferruginaterga Pham, Broad \& Wägele, 2013 Theronia ferruginaterga Pham, Broad \& Wägele, 2013. Journal of Natural History, 47 (23-24): 1525. Holotype: , Vietnam: Kon Tum, Chu Mom Ray NP (RMNH).

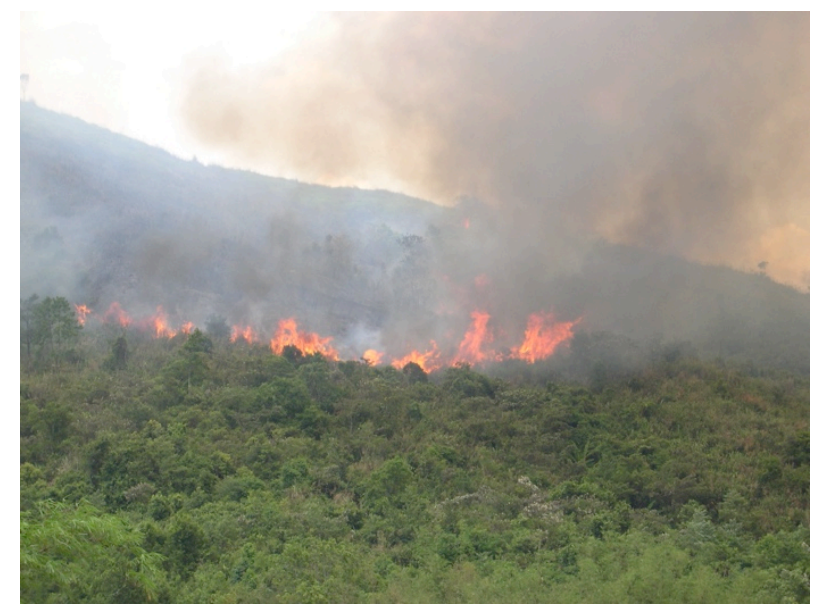

Distribution: Vietnam (Hoa Binh, Thua Thien-Hue, Dak Lak) (Pham et al., 2013b).

Theronia longihastata PHAM, BROAD \& WÄGELE, 2013

Theronia longihastata Pham, Broad \& Wägele, 2013. Journal of Natural History, 47 (23-24): 1528. Holotype: +, Vietnam: Dak Lak, Chu Yang Sin NP (RMNH).

Distribution: Vietnam (Dak Lak) (Pham et al, 2013b).

Theronia tahuythinhi PHAM, BROAD \& WÄGELE, 2013

Theronia tahuythinhi Pham, Broad \& Wägele, 2013. Journal of Natural History, 47 (23-24): 1532. Holotype: q, Vietnam: Dak Lak, Chu Yang Sin NP (RMNH).

Distribution: Vietnam (Dak Lak) (Pham et al, 2013b).

\section{Theronia zebra (VOLLENHOVEN, 1879)}

Pimpla zebra Vollenhoven, 1879. Stett. Ent. Ztg. 40: 147. Holotype: + , Indonesia: Java, Ambarawa (RMNH).

Theronia zebra irididennis Cameron, 1907. Tijdschr. Ent. 50: 99. Holotype: ${ }^{\lambda}$, India: Sikkim (BMNH).

Distribution: Vietnam (Cao Bang, Son La, Hanoi, Vinh Phuc, Phu Tho, Hoa Binh, Nghe An, Ha Tinh, Thua Thien-Hue, Gia Lai), Brunei, China, India, Indonesia, Malaysia, Myanmar, Singapore, Sri Lanka, Taiwan, Thailand and the Philippines (Gupta, 1962; Yu et al., 2005; Pham et al, 2013b).

\section{Discussion}

Although the Central Highlands is recognized as a biodiversity hotspot of Vietnam with a high level of local endemism (Sterling et al., 2006), the fauna and flora of this region are seriously threatened by anthropogenic hazards such as habitat destruction (e.g., mining activities, forest burning, conversion of forest land into industrial plantation and agricultural field, timber logging, dam constrution), illegal poaching and water pollution (see Fig. 2). A long-term strategy for biodiversity conservation of the Central Highlands is urgently needed, particularly in protected areas.

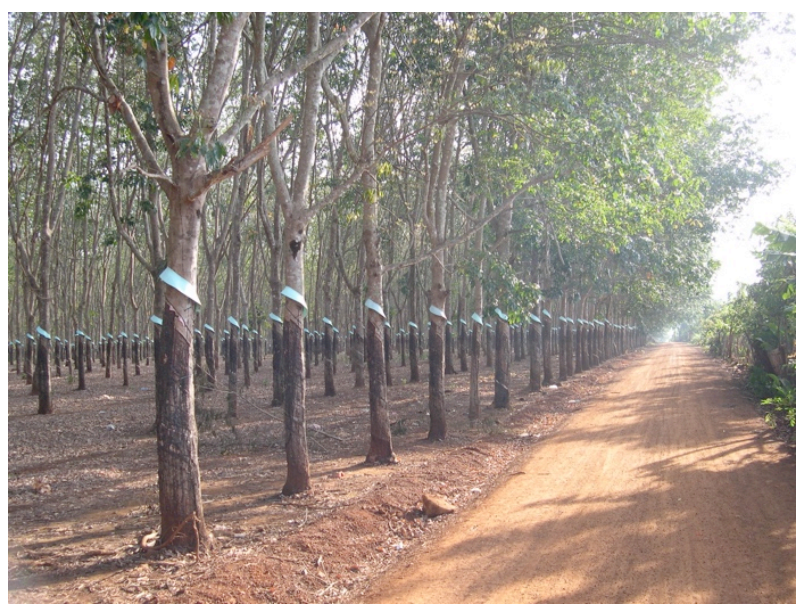

Figure 2. Human impacts (forest fire and rubber plantation) in Gia Lai Province, Vietnam (Photographs by T.V. Hoang) 


\section{References}

[1] Gauld, I.D. \& Dubois, J. (2006) Phylogeny of the Polysphincta group of genera (Hymenoptera: Ichneumonidae; Pimplinae): a taxonomic revision of spider ectoparasitoids. Systematic Entomology, 31: 529-564.

[2] Gauld, I.D., Wahl, D.B. \& Broad, G.R. (2002) The suprageneric groups of the Pimplinae (Hymenoptera: Ichneumonidae): a cladistic re-evaluation and evolutionary biological study. Zoological Journal of the Linnean Society, 136: 421-485.

[3] Gupta, V. K. (1962) Taxonomy, zoogeography, and evolution of Indo-Australian Theronia (Hymenoptera: Ichneumonidae). Pacific Insects Monograph, 4: $1-142$.

[4] Palacio, E., Sääksjärvi, I. \& Vahtera, V. (2007) Lamnatibia, a new genus of the Polysphincta group of genera from Colombia (Hymenoptera: Ichneumonidae; Pimplinae). Zootaxa, 1431: 55-63.

[5] Pham, N.T. (2013). Taxonomy and distributional pattern of Pimplinae (Hymenoptera: Ichneumonidae) from Vietnam. PhD dissertation, $287 \mathrm{pp}$.

[6] Pham, N.T. \& Khuat, L.D. (2008) A species of Xanthopimpla de Saussure, 1892 (Hymenoptera: Ichneumonidae) newly recorded for the ichneumonid fauna of Vietnam. Proceedings of the $6^{\text {th }}$ Vietnam National Conference on Entomology. Agricultural Publishing House, Hanoi: 234-237 (in Vietnamese).

[7] Pham, N.T. \& Le, X.H. (2007) Species composition of the genus Xanthopimpla de Saussure, 1892 (Hymenoptera, Ichneumonidae, Pimplinae, Pimplini) in the Pu Mat National Park, Nghe An Province. Journal of Biology, 29(1): 14-19 (in Vietnamese).

[8] Pham, N.T., Broad, G.R., Dang, H.T. \& Böhme, W. (2013a). A review of the genus Pimpla Fabricius, 1804 (Hymenoptera: Ichneumonidae: Pimplinae) from Vietnam with description of two new species. Organisms Diversity \& Evolution, 13(3): 397-407.

[9] Pham, N.T., Broad, G.R. \& Lampe, K.-H. (2010) Descriptions of two new species of Augerella Gupta (Hymenoptera: Ichneumonidae: Pimplinae) and the first record of $A$. orientalis (Gupta) from Vietnam. Zootaxa, 2654: 17-29.

[10] Pham, N.T., Broad G.R. \& Lampe, K.-H. (2011a) Descriptions of two new species of Augerella Gupta (Hymenoptera: Ichneumonidae: Pimplinae) from Vietnam. Zootaxa, 2745: 68.

[11] Pham, N.T., Broad, G.R., Matsumoto, R. \& Böhme, W. (2012a) First record of the genus Acrodactyla Haliday (Hymenoptera: Ichneumonidae: Pimplinae) from Vietnam, with descriptions of six new species. Zootaxa, 3207: 40-53.

[12] Pham, N.T., Broad G.R., Matsumoto R. \& Wägele W.J. (2011b) Two new species of the genus Chablisea Gauld et Dubois (Hymenoptera: Ichneumonidae: Pimplinae) from Vietnam. Biologia 66 (6): 1134-1139.

[13] Pham, N.T., Broad G.R., Matsumoto R. \& Wägele W.J. (2011c) Revision of the genus Xanthopimpla Saussure (Hymenoptera: Ichneumonidae: Pimplinae) in Vietnam, with descriptions of fourteen new species. Zootaxa, 3056: $67 \mathrm{pp}$.

[14] Pham, N.T., Broad G.R. \& Wägele, W.J. (2011d) The genus Acropimpla Townes (Hymenoptera: Ichneumonidae: Pimplinae) in Vietnam, with descriptions of three new species. Zootaxa, 2921: 1-12.

[15] Pham, N.T., Broad, G.R., Wägele, W.J. (2012c) A review of the genus Camptotypus Kriechbeumer (Hymenoptera, Ichneumonidae, Pimplinae) in Vietnam with description of a new species. Deutsche Entomologische Zeitschrift, 59(1): 129-137.

[16] Pham, N.T., Broad, G.R., Wägele, W.J. (2013b). A review of the Theronia genus-group (Hymenoptera: Ichneumonidae: Pimplinae) from Vietnam with descriptions of five new species. Journal of Natural History, 47(23-24): 1501-1538.

[17] Pham, N.T., Broad, G.R., Wägele, W.J. (2013c). First record of the genus Zaglyptus Foerster (Hymenoptera: Ichneumonidae: Pimplinae) from Vietnam, with description of one new species. Zootaxa, 3702(5): 483-492.

[18] Pham, N.T., Khuat, L.D. \& Dang, H.T. (2011e) New records of Pimplinae (Hymenoptera: Ichneumonidae) from Vietnam. Proceedings of the $7^{\text {th }}$ Vietnam National Conference on Entomology. Agricultural Publishing House, Hanoi: 210-217.

[19] Plant Protection Research Institute (1976) Results of investigation of insects, period 1967-1968 in Vietnam. Agricultural Publishing House, Hanoi: 1-579 pp (in Vietnamese).

[20] Sterling, E.J., Hurley, M.M. \& Le, D.M. (2006) Vietnam: A Natural History. Yale University Press, New Haven and London: 423 pp.

[21] Townes, H.K. \& Chiu, S.C. (1970) The IndoAustralian species of Xanthopimpla (Ichneumonidae). Memoirs of the American Entomological Institute, 14: 1-372.

[22] Yu, D.S., van Achterberg, K. \& Horstmann, K. (2005) World Ichneumonoidea 2004 - Taxonomy, Biology, Morphology and Distribution. DVD/CD. Taxapad, Vancouver, Canada. 